\title{
BMJ Open Characteristics and outcomes of physical activity interventions for individuals with mild traumatic brain injury: a scoping review protocol
}

\author{
Christophe Alarie, ${ }^{1}$ Isabelle J Gagnon, ${ }^{2,3}$ Enrico Quilico, ${ }^{4}$ Bonnie Swaine ${ }^{1,5}$
}

To cite: Alarie C, Gagnon IJ, Quilico $\mathrm{E}$, et al. Characteristics and outcomes of physical activity interventions for individuals with mild traumatic brain injury: a scoping review protocol. BMJ Open 2019;9:e027240. doi:10.1136/ bmjopen-2018-027240

- Prepublication history and additional material for this paper are available online. To view these files, please visit the journal online (http://dx.doi org/10.1136/bmjopen-2018027240).

Received 12 0ctober 2018 Revised 19 February 2019 Accepted 23 May 2019
Check for updates

(C) Author(s) (or their employer(s)) 2019. Re-use permitted under CC BY-NC. No commercial re-use. See rights and permissions. Published by BMJ.

For numbered affiliations see end of article.

Correspondence to

Dr Bonnie Swaine;

bonnie.swaine@umontreal.ca

\section{ABSTRACT}

Introduction Traumatic brain injury (TBI) is a major public health problem, and it is estimated that $85 \%$ of TBls are diagnosed as mild and are commonly referred to as a concussion. In adults, symptoms are expected to resolve within 10-14 days after the injury, but up to $15 \%$ of individuals continue to have symptoms beyond this period. Recent clinical recommendations suggest the use of physical activity (PA) as a therapy to manage persisting symptoms. However, the recommendations regarding PA lack clarity about important intervention parameters to help clinicians deliver the intervention. The objectives of this scoping review are thus to identify the characteristics, the measurement tools, the health-related outcomes and the reported effectiveness of PA-based interventions for adults with persisting symptoms of a mild TBI (mTBI). Methods and analysis This scoping review protocol will follow Arksey and 0'Malley's six-step iterative process enhanced by another study and will be conducted by a team of researchers and clinical experts. Five databases (MEDLINE, CINAHL, PsycINFO, SPORTDiscuss and Embase), as well as Google, will be searched using an extensive search strategy to capture relevant scientific and grey literature. Articles will be selected if they report on an intervention designed to have an impact on health-related outcomes or participation among individuals having sustained an mTBI. A data extraction form based on the Consensus on Exercise Reporting Template and the Template for Intervention Description and Replication checklists will be created. Quantitative and qualitative data will be analysed accordingly, synthesised and collated in tables.

Ethics and dissemination This scoping review generates new knowledge from published and publicly available literature; thus, an ethical approval is unnecessary to conduct this research. Dissemination of the results will involve all team members in activities aimed to facilitate knowledge uptake among TBI rehabilitation clinical experts locally, nationally and internationally.

\section{INTRODUCTION}

Traumatic brain injury (TBI) is a major public health problem, and it is estimated that each year, more than 10 million individuals worldwide will experience a TBI that may result in either mortality or hospitalisation. ${ }^{1} \mathrm{Up}$ to

\section{Strengths and limitations of this study}

- This will be the first scoping review to critically appraise the characteristics of physical activity (PA)-based interventions designed to improve health-related outcomes in adults with persistent symptoms post mild traumatic brain injury.

- Clinical partners will be integrated into the research process in a creative yet feasible way to ensure enhanced interpretation and better applicability of the results.

- The combination of two expert consensus-based checklists (Consensus on Exercise Reporting Template and Template for Intervention Description and Replication) to guide the data extraction will allow better identification of key characteristics of PA-based interventions.

- Although this study aims to describe the characteristics of PA-based interventions, it will not allow inferences about the effectiveness of these parameters, which could be further investigated in a systematic review.

$85 \%$ of TBIs are diagnosed as mild traumatic brain injury $(\mathrm{mTBI})^{2}$ and are commonly referred to as a concussion. ${ }^{3}$ A wide range of consequences may result from mTBI (eg, headaches, anxiety, difficulty concentrating, fatigue and sleep disturbances) ${ }^{4}$ which, in turn, may limit the individual's activities, restrict their participation and decrease their quality of life. ${ }^{5}$

The symptoms of mTBI typically subside within 10-14 days after the injury in adults. ${ }^{4}$ An individual who fails to recover completely within this time frame is considered to have persisting symptoms, ${ }^{4}$ and it is likely that $26 \%$ of individuals who sustain an mTBI will have persisting symptoms beyond 3 months. ${ }^{67}$ Interventions aiming to reduce persisting physical and cognitive symptoms are critical in order to return to preinjury functioning. ${ }^{89}$

Clinical practice guidelines (CPGs) aim to improve the quality and decrease the 
variability of healthcare services by providing clinical experts key evidence-based recommendations to implement within their practices. ${ }^{10}$ Based on the highest available evidence, CPGs for the management of adults with persisting symptoms of mTBI were developed to support clinical decision-making and improve rehabilitation outcomes. The Ontario Neurotrauma Foundation's CPG for adults with persisting symptoms of $\mathrm{mTBI}$ and the CPG for military personnel with mTBI produced by the American Department of Defense and Veterans Affairs both recommend using physical activity (PA) as a therapy to alleviate mTBI-related symptoms (eg, headache, fatigue or sleep disturbances) and to improve mood, health status and exercise tolerance. ${ }^{611}$ These CPGs corroborate recommendations from the latest Consensus Statement on Concussion in Sport, which suggests including a symptom-limited, progressive exercise intervention for individuals who experience persisting symptoms ( $>1$ month) after mTBI. ${ }^{4}$ Collectively, these recommendations promote the use of PA as an intervention that can help decrease and manage prolonged persistent symptoms of mTBI in adults.

These CPGs and consensus statements are fairly recent, but they lack specific information about how PA interventions should be delivered by service providers. Important PA intervention characteristics, such as frequency, intensity, time, type of exercise and progression patterns, are missing. This lack of clear parameters leaves clinicians using trial-and-error methods instead of an evidencebased approach. Indeed, the complexity and the lack of applicability of recommendations are CPG-related barriers to the implementation and use of evidence-based recommendations. ${ }^{12}$ Insufficient information about PA interventions leaves many clinical questions unanswered: Should the PA intervention be delivered in a group or individually? Should PA adherence be measured and, if yes, how? Service providers who apply recommendations from CPGs also require assessment tools to evaluate the health-related outcomes of PA interventions. For example, with the exception of the postconcussion symptoms scale in the CPGs, it is unclear which clinical tool should be used to measure the effectiveness of a PA intervention. Clear parameters that guide PA interventions may promote optimal dosage and type of planned PA in order to maximise benefits and accommodate individual preferences through different activities. ${ }^{13}$

The primary objective of this scoping review is to identify characteristics of PA-based interventions available in the scientific and grey literature designed to improve health-related outcomes in adults with persistent symptoms of an mTBI and to report on the intervention's effectiveness, if available. The secondary objectives are to document the health-related outcomes and the measurement tools related to PA interventions found in the literature. This information could help researchers and healthcare providers select appropriate outcomes and outcome measurement tools for future research or PA programme design and implementation.

\section{METHODS AND ANALYSIS}

\section{Protocol}

This scoping review will follow the six-step iterative framework of Arksey and O'Malley, which was later enhanced by Levac et al in 2010 to ensure structure and rigour during a thorough investigation of the scientific and grey literature. ${ }^{14}{ }^{15}$ This work will be conducted by a team of two doctoral students who are supervised by two rehabilitation scientists and assisted by four clinical experts and administrators from a specialised mTBI rehabilitation programme (administrator, clinical coordinator, kinesiologist and physiotherapist). The multidisciplinary clinical team was involved in the design of the study and will participate in multiple key steps of this review as described below. This collaborative approach is a creative yet feasible way to involve clinical partners, as well as to ensure an accurate interpretation of the review results and their applicability in the clinical setting. To better report this scoping review protocol, the authors used the Preferred Reporting Items for Systematic Reviews and Meta-Analyses (PRISMA) Protocols reporting guidelines and the PRISMA Extension for Scoping Reviews for scoping review extension. ${ }^{16} 17$

\section{Step 1: Identifying the research question}

A preliminary consultation with the clinical partners led to the development of an initial research question that was used as a starting point to guide the development of the search strategy: What are the characteristics and health-related outcomes of PA interventions designed for individuals with $m T B I$ ? In the context of this review, we define PA based on a combination of two definitions. The World Health Organization (2018) defines PA as 'any bodily movement produced by skeletal muscles that requires energy expenditure', and the 2007 Oxford dictionary's definition adds, 'Any form of body movement that has a significant metabolic demand. Thus, PAs include training for and participation in athletic competitions, the performance of strenuous occupations, doing household chores, and non-sporting leisure activities that involve physical effort'. ${ }^{18}{ }^{19}$ This definition would refer to different types of activities involving a physical effort, ranging from recreation activities, such as walking slowly, to high-intensity aerobic training. Knitting in a chair would not be considered a PA in the scoping review. The research question is subject to change during the process as new questions and reflections might emerge during each iterative step of the scoping review.

\section{Step 2: Identifying relevant studies}

Literature will be selected if it reports on a PA-based intervention, provided in any setting (eg, inpatient or outpatient rehabilitation) or in the community, designed to impact on health-related (physical, mental or psychosocial) outcomes or participation in individuals of all ages having sustained any severity of TBI. However, at least one participant in the study sample must have sustained an mTBI. Moreover, the intervention may target persons 
with all types of injury severity, but it must be pertinent for persons with mTBI. For example, interventions such as constraint-induced movement therapy is indicated for an individual with a motor impaired upper extremity and not particularly for someone with an mTBI. ${ }^{20}{ }^{21}$ Consequently, articles on this intervention would be excluded. Articles with participants of all ages will be included because the age range of subjects in paediatric studies can include teenagers, which might overlap with young adults.

PA interventions are used in many fields of research (ie, rehabilitation sciences, education, psychology and exercise sciences) and thus can be presented in many formats. Consequently, the literature search will cover published and unpublished literature (grey literature), including original research, theses and books. A broad yet feasible range of five databases (MEDLINE, CINAHL, PsycINFO, SPORTDiscuss and Embase) will be accessed using an extensive search strategy validated by a specialised university librarian. Keywords related to TBI and PA corresponding to subject headings (or Medical Subject Headings) will be included in the search strategy (eg, Brain Injuries, Traumatic, Brain Concussion, Exercise Therapy and Exercise). Other relevant keywords will be added to the search strategy to enhance the strategy and will be used to search titles, abstracts and subjects of references contained in the databases (eg, mild traumatic brain injury, physical fitness and motor activity). The search strategy is provided in the supplementary file (online supplementary file I). Through an iterative and concerted process, analysis of the search results and retrieved articles will guide the refinement of the search strategy to achieve a balance between feasibility and breadth. Opinion articles, posters, oral presentations and abstracts from conferences will be excluded because they may lack explicit information about reported interventions. Systematic reviews will be excluded, but their reference lists will be examined by the authors to ensure relevant articles are retained. Animal model studies will also be excluded due to their lack of applicability to human study contexts.

The grey literature search will be conducted on Google using a modified version of the final search strategy to find TBI-related PA interventions described in other formats, such as PDF documents, books and websites in the first 10 pages of results (approximately 100 results). Moreover, a hand search of reference lists of all selected documents will be performed to ensure that all key studies are captured. Each added reference to the initial search will be documented and will be reported in a PRISMA flowchart created for this study. ${ }^{22}$ Languages will be restricted to both French and English, as authors are fluent in both languages. Searches will be limited to published literature after 1990, when recommendations about PA were first established. ${ }^{23}$ Results will be managed using reference manager software (EndNote), and duplicates will be removed before selection.

\section{Step 3: Study selection}

Study selection will be conducted by two independent reviewers in two subsequent phases: (1) abstract and title review and (2) full-text review. Based on initial eligibility criteria, the reviewers will start to examine/discuss a random sample of 100 retrieved references to determine whether the article should be considered, rejected or if they are unsure. Inter-rater reliability (IRR) will be computed with a three-level kappa ( $\kappa)$ statistic. As needed, the eligibility criteria will be discussed by the researchers and modified for more clarity. This initial selection process will be repeated with a sample of 300-500 references until the agreement between the two reviewers reaches a mean $\kappa>0.75(\kappa>0.75=$ excellent agreement $) .{ }^{24}$ When acceptable agreement is achieved, the reviewers will independently assess the remaining articles. They will also meet at the midpoint and endpoint of remaining articles to discuss any changes, thoughts or needs for clarification. The fulltext review phase will follow the same rigorous method in order to determine IRR. This time, 10-20 articles will be randomly selected and cross-examined by the same two reviewers, and then re-examined independently until they reach excellent agreement or a mean $\kappa>0.75$. If a disagreement cannot be resolved through consensus in any of the two phases, a third independent reviewer will be consulted. Reasons for excluded articles during the second phase will be reported in the PRISMA flowchart. As the selection unfolds, criteria can be refined or clarified if needed, and if a criterion is modified at a later stage of the article selection, authors will ensure that the previous steps will comply with the change and report the changes in the PRISMA flowchart.

\section{Step 4: Charting the data}

A preliminary data extraction form will be created in an Excel spreadsheet based on the combination of the 12-item Template for Intervention Description and Replication checklist (TIDieR) and the 16-item Consensus on Exercise Reporting Template (CERT) checklist. ${ }^{25} 26$ Both checklists were systematically developed to improve the quality of reporting interventions in rehabilitation sciences. However, the CERT includes specific key items to better report an exercise programme (eg, motivation strategies, decision rules for determining exercise progression and decision rules to describe the starting level). Descriptive quantitative data about the number, the age and the gender of participants with an mTBI included in each article will be extracted. More qualitative information related to each item of the extraction form will be extracted from each selected article. For example, all information related to the type of exercise equipment (CERT item 1), a home programme (CERT item 8), description of the exercise intervention (CERT item 13), the setting in which the exercises are performed (CERT item 12) or about the extent to which the intervention was delivered as planned (CERT item 16) will be extracted. If no information was provided about a specific item in an article, it will also be noted and compiled. 
CERT was designed to be used in conjunction with the TIDieR checklist. Due to the overlap of items from both checklist information, only two items from the TIDIER will be included in the data extraction form (item 1: name of the intervention and item 2: rationale, theory or goals of the intervention).

The extraction form will also consist of other categories including, but not limited to, primary and secondary outcomes, measurement tools and effectiveness. The clinical partners will validate this extraction form during a second consultation, and additional categories may be included during the iterative process if deemed appropriate by the team.

Data will be extracted from the selected articles and tabulated by two independent reviewers. A sample of five studies will be extracted by each reviewer and then compared during a work session to ensure compatibility between extraction methods and to enhance the extraction form, with new or more precise categories if needed. The extraction team will repeat this process until the extractors/reviewers agree that they consistently assess and extract information from each article in a compatible way. Then, reviewers will meet regularly (eg, every 10-20 articles) to address any challenges and to ensure concordance with their reporting methods.

\section{Step 5: Collating, summarising and reporting the results}

Analyses of the quantitative and qualitative data will be performed by the researchers. Quantitative data such as numerical descriptive characteristics of PA interventions (eg, year of publication, age and number of mTBI individuals in the study, number of interventions using motivation strategies) will be summarised into tables. In addition, selected articles reporting on PA interventions will be carefully assessed with the CERT checklist assessment form. ${ }^{26}$ Each of the checklist's 16 items will be categorised as yes if the information was provided or no if the information is missing. Following a similar process for reliability, two independent reviewers will assess a small subset of articles and will compare their results. Discrepancies in assessment will be resolved through discussion, and this step will be repeated until reviewers reach an excellent IRR of $\kappa>0.75$. Then, the first author will assess the remaining articles. Qualitative data will be synthesised and collated in tables. Quantitative results may be presented graphically (eg, number of PA interventions per study per year and percentage of types of interventions) and qualitative results may be presented narratively and/or in tables. The different PA characteristics and key PA principles will be summarised and reported in multiple matrices. Outcome constructs and measurement tools will be reported and summarised in tables. Measurement tools used in the different studies/articles will also be aggregated into categories and summarised in tables.

\section{Step 6: Consultation with stakeholders}

The clinical experts mentioned above will be consulted throughout the review process (ie, prior to the development of the study to define the research question, and while designing the research protocol to validate and possibly enhance the data extraction form). Consultation will also occur at the end of the review to assist with the interpretation of the results in order to improve their clinical relevance and to determine the best ways to mobilise the knowledge generated by the review.

\section{Patient and public involvement}

Patients and the public will not be involved in this scoping review.

\section{ETHICAL CONSIDERATIONS AND DISSEMINATION}

A scoping review generates new knowledge from published and publicly available literature and does not involve human participants. Therefore, a research ethics board approval is unnecessary to conduct this research. Although our clinical partners will be involved in multiple steps of the study, they are primarily involved as expert consultants, and their input may deepen the understanding and enhance the scope of the results. Members of the group will work together during work sessions to co-create a final document that will be used to help disseminate the results of this review to other clinicians working in mTBI rehabilitation. Dissemination of the results will involve all team members through regional, national and international scientific and clinical activities and conferences, the publication of a manuscript, and other activities aimed to generate awareness and increase knowledge uptake of mTBI rehabilitation clinical experts.

\section{DISCUSSION}

The results of this scoping review will provide detailed information about the state of the existing literature regarding the important characteristics, intervention parameters and tools to measure health-related outcomes of PA-based interventions designed for adults with persistent symptoms of mTBI. These results may assist clinical experts with the use of PA in the management of adults with mTBI and may ultimately improve patient outcomes. Moreover, the results of this scoping review will inform researchers about the effectiveness of multiple PA parameters, which may be further investigated in a systematic review.

\section{Author affiliations}

'École de Réadaptation, Université de Montréal, Montréal, Québec, Canada ${ }^{2}$ School of Physical and Occupational Therapy, McGill University, Montréal, Québec, Canada

${ }^{3}$ Department of Trauma, Montreal Children's Hospital of the McGill University Health Center, Montréal, Québec, Canada

${ }^{4}$ Rehabilitation Science Institute, University of Toronto, Toronto, Canada

${ }^{5}$ Centre de recherche interdisciplinaire en réadaptation du Montréal metropolitain, Montréal, Québec, Canada

Acknowledgements We thank the clinical partners, Pierre Vincent, Geneviève Léveillé, Geneviève Lagarde, Julie Brière , Manh -Tien Thierry Nguyen, Louise 
Chartier and Pierre Goulet, for their involvement in the identification of the research question and future involvement in the consultation steps.

Contributors All authors designed the protocol, reviewed the manuscript, approved the final version and participated in the six steps. CA drafted the manuscript.

Funding This work is supported by the Edith Strauss Rehabilitation Research Projects Foundation. This grant provides salary support to $C A$ and $E Q$.

Competing interests None declared.

Patient consent for publication Not required.

Provenance and peer review Not commissioned; externally peer reviewed.

Open access This is an open access article distributed in accordance with the Creative Commons Attribution Non Commercial (CC BY-NC 4.0) license, which permits others to distribute, remix, adapt, build upon this work non-commercially, and license their derivative works on different terms, provided the original work is properly cited, appropriate credit is given, any changes made indicated, and the use is non-commercial. See: http://creativecommons.org/licenses/by-nc/4.0/.

\section{REFERENCES}

1. Hyder AA, Wunderlich CA, Puvanachandra $P$, et al. The impact of traumatic brain injuries: a global perspective. NeuroRehabilitation 2007;22:341-53.

2. Feigin VL, Theadom A, Barker-Collo S, et al. Incidence of traumatic brain injury in New Zealand: a population-based study. Lancet Neurol 2013;12:53-64.

3. Marshall S, Bayley M, McCullagh S, et al. Updated clinical practice guidelines for concussion/mild traumatic brain injury and persistent symptoms. Brain Inj 2015;29:688-700.

4. McCrory P, Meeuwisse W, Dvorak J, et al. Consensus statement on concussion in sport-the 5 th international conference on concussion in sport held in Berlin. Br J Sports med 2016:1-10.

5. Perroux M, Lefebvre H, Levert M-J, et al. Besoins perçus et participation sociale des personnes ayant un traumatisme crânien léger. Santé Publique 2013;25:719-28.

6. Ontario Neurotrauma Foundation. Guideline For Concussion/mild Traumatic Brain Injury \& Persistent Symptoms 3Rrd Edition, For Adults Over 18 Years of Age. 2018. http://braininjuryguidelines.org/ concussion/index.php?id=1.

7. Cassidy JD, Cancelliere C, Carroll LJ, et al. Systematic review of selfreported prognosis in adults after mild traumatic brain injury: results of the International Collaboration on Mild Traumatic Brain Injury Prognosis. Arch Phys Med Rehabil 2014;95:S132-S151.

8. Makdissi M, Schneider KJ, Feddermann-Demont N, et al. Approach to investigation and treatment of persistent symptoms following sport-related concussion: a systematic review. $\mathrm{Br} J$ Sports Med 2017;51:958-68.
9. Schneider KJ, Leddy JJ, Guskiewicz KM, et al. Rest and treatment/ rehabilitation following sport-related concussion: a systematic review. Br J Sports Med 2017;51:930-4.

10. Lohr KN, Field MJ. Guidelines for clinical practice: from development to use. Washington: WASH DC, National Academies Press, 1992.

11. Department of Veterans Affairs, Department of Defense. VA/ DoD clinical practice guideline for management of concussion/ mild traumatic brain injury. 2016 https://www.healthquality.va.gov/ guidelines/Rehab/mtbi/ (Accessed oct 2018).

12. Fischer $\mathrm{F}$, Lange $\mathrm{K}$, Klose $\mathrm{K}$, et al. Barriers and strategies in guideline implementation - a scoping review. Health Care 2016;4:36.

13. Ammann $\mathrm{BC}$, Knols $\mathrm{RH}$, Baschung $\mathrm{P}$, et al. Application of principles of exercise training in sub-acute and chronic stroke survivors: a systematic review. BMC Neurol 2014;14:167-78.

14. Arksey H, O'Malley L. Scoping studies: towards a methodological framework. Int J Soc Res Methodol 2005;8:19-32.

15. Levac D, Colquhoun H, O'Brien KK. Scoping studies: advancing the methodology. Implement Sci 2010;5:69-78.

16. Moher D, Shamseer L, Clarke M, et al. Preferred reporting items for systematic review and meta-analysis protocols (PRISMA-P) 2015 statement. Syst Rev 2015;4:1.

17. Tricco AC, Lillie E, Zarin W, et al. PRISMA Extension for Scoping Reviews (PRISMA-ScR): Checklist and Explanation. Ann Intern Med 2018;169:467-73.

18. World health organisation. Global strategy on diet, physical activity and health. 2018 https://www.who.int/dietphysicalactivity/pa/en/ (Accessed oct 2018).

19. Kent M. Oxford dictionary of sports science and medicine. J Sports Sci Med 2007;6:152.

20. Sirtori V, Corbetta D, Moja L, et al. Constraint-induced movement therapy for upper extremities in stroke patients. Cochrane Database Syst Rev 2009;7.

21. Hoare BJ, Wasiak J, Imms C, et al. Constraint-induced movement therapy in the treatment of the upper limb in children with hemiplegic cerebral palsy. Cochrane Database Syst Rev 2007;113.

22. Moher D, Liberati A, Tetzlaff J, et al. Preferred reporting items for systematic reviews and meta-analyses: the PRISMA statement. Ann Intern Med 2009;151:264-9.

23. Pollock MLP, Froelicher V. American College of Sports Medicine position stand. The recommended quantity and quality of exercise for developing and maintaining cardiorespiratory and muscular fitness in healthy adults. Med Sci Sports Exerc 1990;22:235-45.

24. Orwin RG, Cooper IH, Hedges LV, et al. The handbook of research synthesis. New York, NY: Russell Sage Foundation, 1994:139-62.

25. Hoffmann TC, Glasziou PP, Boutron I, et al. Better reporting of interventions: template for intervention description and replication (TIDieR) checklist and guide. BMJ 2014;348:g1687.

26. Slade SC, Dionne CE, Underwood M, et al. Consensus on Exercise Reporting Template (CERT): Explanation and Elaboration Statement. Br J Sports Med 2016;50:1428-37. 